\title{
Floodplain Evaluation Matrix (FEM) - Eine umfassende Methode zur Bewertung von Überflutungsräumen im Rahmen eines integrierten Hochwasserrisikomanagements
}

\author{
Bernhard Schober · Christoph Hauer · Helmut Habersack
}

Online publiziert: 28. November 2017

(c) Der/die Autor(en) 2017. Dieser Artikel ist eine Open-Access-Publikation.

\begin{abstract}
Zusammenfassung Der Schutz - und wo möglich die Wiederherstellung - von Überflutungsflächen im Rahmen eines integrierten Hochwasserrisikomanagements wird seit Jahren von höchster

Floodplain Evaluation Matrix (FEM) - A holistic method for evaluating floodplains in the context of integrated flood risk management
\end{abstract} Ebene als nachhaltige und nicht-strukturelle Maßnahme gefordert. Dennoch steht dem in der Realität ein fortschreitender Flächenverbrauch für Siedlungen und Infrastruktur insbesondere auf flussnahen Flächen gegenüber. Die hier vorgestellte Floodplain Evaluation Matrix (FEM) stellt eine Methode dar, mit der es möglich wird, den Wert von zusammenhängenden Überflutungsflächen festzustellen und Grenzwerte zu definieren, die eine fortschreitende Verbauung und damit den Summationseffekt hintanhalten. Basierend auf der Analyse verschiedener hydrologischer und hydraulischer Parameter wird eine Bewertung jeder Fläche vorgenommen und deren Wirksamkeit quantifiziert, um diese folglich auf Basis national einheitlicher Grenzwerte im Wasserrechtsverfahren aber auch mit geeigneten Mitteln der Raumordnung und des Baurechts freihalten zu können.

DI B. Schober $(\bowtie)$.

PD DI Dr. C. Hauer .

Univ.-Prof. DI Dr. H. Habersack

Department für

Wasser-Atmosphäre-Umwelt (WAU)

Institut für Wasserwirtschaft,

Hydrologie und konstruktiver

Wasserbau (IWHW), Christian

Doppler Labor für Sedimentforschung

und -management, Universität

für Bodenkultur Wien,

Muthgasse 107, 1190 Wien, Österreich

bernhard.schober@boku.ac.at
Abstract The preservation - and where possible the restoration - of floodplains within an integrated flood risk management is a high-level demand for many years now and is considered as sustainable and non-structural measure. However, in reality land consumption for settlements and infrastructure is still ongoing at a very high rate especially on river floodplains. This paper presents the Floodplain Evaluation Matrix (FEM) - a novel approach which enables to assess the effectiveness of coherent floodplains and to define threshold values in order to impede progressing construction activities and the associated stepby-step loss of floodplain effectiveness due to summation effects. Based on the analysis of different hydrological and hydraulic parameters, each distinct floodplain is evaluated and its effectiveness is quantified. This provides the basis for the preservation of floodplains by means of nationwide valid threshold values of the water rights act but also within the fields of spatial planning and building laws.

\section{Einleitung}

Weltweit gesehen sind Hochwässer für ein Drittel aller katastrophenbedingten Schäden (Schäden an Gebäuden, Infrastruktur und landwirtschaftlichen Kulturen) sowie für die Hälfte aller durch Naturkatastrophen getöteten Personen verantwortlich (Koirala 2011). Internationale Studien in diesem Kontext haben gezeigt, dass die Zahl der Überflutungen in Europa seit der Jahrtausendwende stark angestiegen ist, was ebenfalls zu einer starken Erhöhung der wirtschaftlichen Schäden geführt hat (Barredo 2007). In Österreich ha- ben die Großüberflutungsereignisse von 2002 (9 Todesopfer; geschätzter Schaden von 2 bis 3 Mrd. €; Habersack und Moser 2003), 2005 (5 Todesopfer, ca. 550 Mio. $€$ Schaden; Habersack et al. 2009) und 2013 (ca. 866 Mio. $€$ Schaden; Habersack et al. 2014a) gezeigt, wie vulnerabel von Menschen genutzte Gebiete in Bezug auf Hochwässer sind. Die hohen Schadenssummen waren zusätzlich auf die transportierten und abgelagerten Schwebstoffe zurückzuführen (vgl. Barsch et al. 1994), die massive Schäden an Gebäuden nach sich zogen (Habersack et al. 2009). Im Jahr 2013 kam es auch durch die eigendynamische Entlandung von Stauräumen an Inn und Donau zu weitreichenden Flurschädigungen durch abgelagerte Schwebstoffe (Flicker und Freiberger 2014). Weiters zeigte sich in den Katastrophenjahren 2002 und 2005, dass bei Ereignissen $>\mathrm{HQ}_{100}$ die überproportional hohe Strömungsenergie zur eigendynamischen Verbreiterung der Fließgewässer führt, einhergehend mit massiven Schäden bis hin zur Zerstörung von Hochwasserschutzmaßnahmen oder den Gewässern angrenzenden Gebäuden (Krapesch et al. 2011).

Ein Grund für den Anstieg von Hochwasserereignissen sind zum einen veränderte Abflussregime (z.B. durch Landnutzungsänderungen im gesamten Einzugsgebiet oder Klimawandel; vgl. Milliman et al. 2008; Vörösmarty et al. 2010) und zum anderen stark veränderte Strukturen der Fließgewässer (wie z.B. Begradigungen, Uferschutzmaßnahmen oder die Abtrennung von Überflutungsräumen; Bogardi et al. 2012; Syvitsky et al. 2005; Walling 2006), welche nicht mehr in der Lage sind, die großen Wassermassen im Ereignisfall schadfrei abzuführen. In diesem Zusammenhang zeigt sich auch die Vulnerabilität konventioneller (struktureller, technischer) Hochwasserschutzmaßnahmen wie z. B. von Hochwasserschutzdeichen/-dämmen (Kundzewicz 
und Menzel 2005; Messner und Meyer 2006; De Kok und Grossmann 2010). Der Ausbau oder die Erhöhung von HW-Dämmen ist hierbei nicht ausreichend, um Schäden zu verhindern, da hierdurch verstärkt Überflutungsflächen vom Fluss abgetrennt werden und somit für Rückhalt und Abfluss verloren gehen und bei Extremereignissen die Gefahr von Verwerfungen im Überlastfall zusätzlich erhöht wird (Hauer und Habersack 2009). Somit wird das verfügbare Retentionsvolumen eines Flusses weiter verringert. Zudem weisen derart (vermeintlich) hochwasserfrei gemachte Gebiete ein höheres Schadenspotenzial auf, da sie Besiedlung und damit höherwertige Landnutzungen anziehen (PenningRowsell und Tunstall 1996; Freshwater und Arthur 1985). Im Falle des Restrisikos (Überströmen der Dämme infolge der Überschreitung des Bemessungsereignisses oder bei Versagen des Schutzbauwerkes durch Dammbruch) treten folglich erheblich größere Schäden als vor der gesetzten Maßnahme auf.

Daher wurde in den letzten Jahren vermehrt Augenmerk auf neue Ansätze im integrierten Hochwasserrisikomanagement gelegt, die nichtstrukturelle Maßnahmen in die konventionellen Konzepte miteinbeziehen (vgl. EU-Hochwasser-Richtlinie 2007/60/EC). Durch diese Kombination soll eine optimale Wirkung und gleichzeitige Kosteneffizienz angestrebt werden (Kundzewicz und Menzel 2005). Ein Hauptanliegen besteht dabei in der Erhaltung und - wo möglich der Wiederherstellung - von Überflutungsflächen, da diese auf der einen Seite als natürliche Rückhalte- und Abflussräume fungieren (auch für transportierte Schwebstoffe) und andererseits durch deren Freihaltung einer Erhöhung des flussnahen Schadenspotenzials entgegengewirkt wird (Blackwell und Maltby 2006; Wharton und Gilvear 2007).

In der Realität zeigt sich jedoch ein starker Nutzungsdruck auf derartige Überflutungsflächen. Schätzungen zufolge dürften weltweit bereits mehr als $50 \%$ der Überflutungsflächen von ihren Flüssen abgetrennt worden sein, wobei dieser Prozentsatz für Europa sicherlich nochmals höher ist (Kundzewicz und Menzel 2005). Nutzungen wie Siedlungen oder Infrastruktur stehen dabei im Gegensatz zu den Forderungen des passiven Hochwasserschutzes.

Um eine ausgewogene und optimierte Nutzung von Überflutungsflächen zu gewährleisten, die sowohl den wirtschaftlichen und siedlungspolitischen Forderungen als auch jenen des Hochwasserschutzes gerecht wird, bedarf es einer Bestandsaufnahme der vorhandenen Überflutungsflächen sowie deren Wirksamkeit in Bezug auf mehrere Parameter. Diese Wirkungsanalyse und die darauf aufbauende Prioritätenreihung von Überflutungsflächen sollte die Grundlage für nicht-strukturelle Hochwasserschutzmaßnahmen im Sinne eines integrierten Hochwasserrisikomanagements darstellen bzw. auch als Grundlage des erweiterten Flussgebietsmanagements (vgl. GERM, Pleschko et al. 2017, in diesem Heft) und von Sedimentbewirtschaftungsmaßnahmen (Verbesserung des Sedimentkontinuums bei Kraftwerken, Hauer et al. 2016) dienen.

\section{Entwicklung der Floodplain Evaluation Matrix (FEM)}

Erste Schritte in Richtung einer integrativen Überflutungsflächenbeurteilung wurden bereits ab dem Jahr 2007 mit dem EU-geförderten ERA-NET CRUEProjekt „Pro_Floodplain“ gesetzt, bei welchem WissenschaftlerInnen und ExpertInnen aus verschiedenen europäischen Ländern einen Kriterienkatalog für die integrative Beurteilung von Überflutungsflächen erarbeiteten (Habersack et al. 2008). Die Methodik wurde folglich in Hinblick auf österreichische Gewässer an mehreren Flüssen mit unterschiedlicher Charakteristik (Ill in Vorarlberg, Inn in Tirol, Lavant in Kärnten, Krems in Oberösterreich und Raab in der Steiermark) erprobt und verfeinert (Habersack et al. 2014b). Das Ergebnis - die integrative Floodplain Evaluation Matrix (FEM) - kombiniert hydrologische und hydraulische Parameter zur objektiven Beurteilung von Überflutungsflächen. Die entwickelte Methode, welche sich auf die wesentlichen Komponenten in den HWAnalysen bezieht und Hydraulik und Hydrologie sowohl einzeln als auch integrativ bewertet, wurde als Grundlage für integratives Flussgebietsmanagement definiert, mit dem Ziel, zukünftig weitere Parameter der Raumnutzung, des Schadenspotenzials, der Gewässerökologie aber auch vor allem des Feststoffhaushalts und der Morphodynamik der Fließgewässer in den integrativen Evaluierungsprozess miteinzubeziehen („extended FEM“).

\section{Methodik}

Die Floodplain Evaluation Matrix (FEM) basiert im Wesentlichen auf zwei Prinzipien (Habersack et al. 2013): i) einem skalenorientierten Ansatz und ii) einer sektoralen Beurteilung unterschiedlicher hydraulischer und hydrologischer Parameter (MultiparameterAnsatz), welche anschließend in einer Gesamtbewertung des jeweiligen Überflutungsraums integrativ zusammengeführt werden.

\subsection{Skalenansatz}

Die Beurteilung von Überflutungsflächen mittels der FEM-Methode erfolgt auf drei verschiedenen räumlichen Ebenen.

Als Mikro-Skala werden die einzelnen Überflutungsflächen selbst verstanden. Die Abgrenzung erfolgt aufgrund geomorphologischer und hydraulischer Kriterien. So können geomorphologisch oder anthropogen bedingte Engstellen sowie Zubringer natürliche Grenzen von Überflutungsflächen darstellen. Besonders wichtig ist, dass Überflutungsräume derart abgegrenzt werden, dass die Fließcharakteristik im jeweiligen Überflutungsraum nicht verfälscht wird. Es ist darauf $\mathrm{zu}$ achten, dass die Auslaufrandbedingungen der Überflutungsflächen so gesetzt und im Modell definiert werden, dass die Abflusscharakteristik jedes Raums gut abgebildet ist. Das bedeutet auch, dass - je nach Situation - Überflutungsflächen nach links- und rechtsufrig abgegrenzt oder gemeinsam als funktionelle Einheit betrachtet werden können. Die Mikro-Skala dient der Ermittlung der Bewertungsparameter für jede einzelne Überflutungsfläche für sich selbst, womit das Potenzial der Fläche ermittelt wird. Dieses ist damit unabhängig von etwaigen Änderungen der flussauf liegenden Flächen und kann auch die tatsächliche Hydrologie widerspiegeln. Durch diese Vorgehensweise wird sichergestellt, dass die Ergebnisse für jede Überflutungsfläche auch der tatsächlich zu betrachtenden (und als rechtsverbindlich anzusehenden) Jährlichkeit (z.B. $H_{100}$ ) für genau diese Überflutungsfläche entsprechen (Anm.: im Gegensatz dazu würde bei großräumigen Modellierungen die Hochwasserwelle durch die Rückhaltewirkung im Längsverlauf des Flusses bereits eine Abminderung erfahren, sodass sie vor allem weiter flussab nicht 


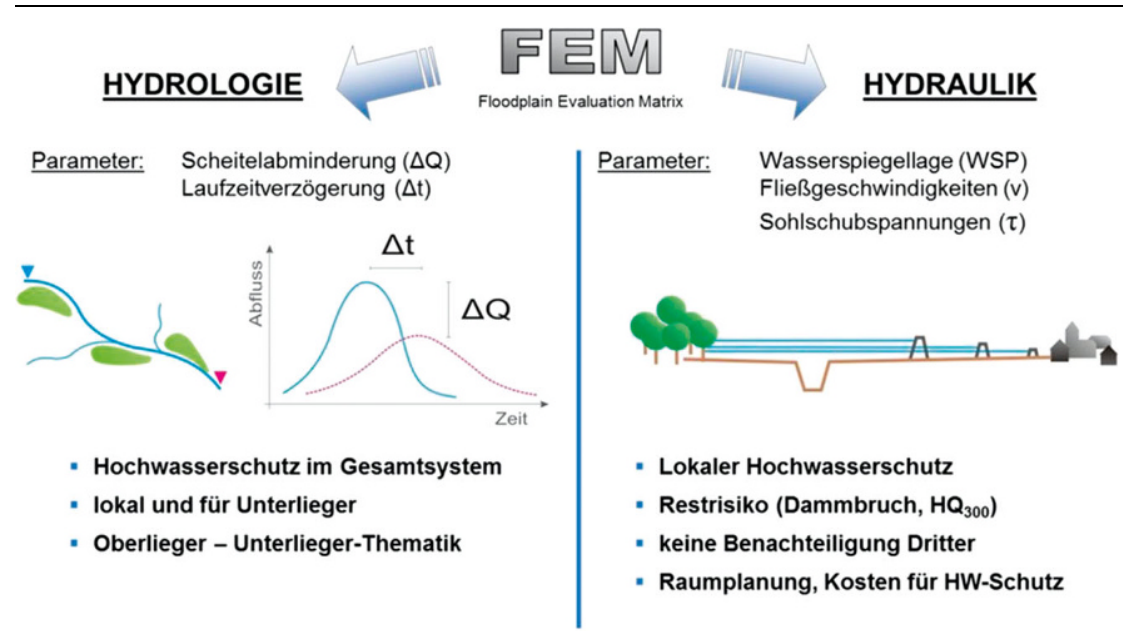

Abb. 1 Multiparameter-Ansatz der FEM

mehr der zu betrachtenden Jährlichkeit entspricht). Dabei wird für jede Überflutungsfläche ein hydrodynamischnumerischer Rechenlauf durchgeführt. Die Ergebnisse sind von anderen Überflutungsflächen unabhängig und somit für einen objektiven Vergleich geeignet.

Dieser Vergleich wird sodann auf Ebene der Meso-Skala durchgeführt. Die Meso-Skala umfasst Flussabschnitte, die sich durch eine einheitliche hydrologische und morphologische Charakteristik auszeichnen. Flussabschnitte sind hierbei so abzugrenzen, dass sie mehrere Kilometer bis zig Kilometer lang sind und eine repräsentative Anzahl von Überflutungsflächen gemäß Abgrenzung auf Ebene der MikroSkala (im Idealfall zwischen 10 und 30) enthalten. Ändert sich im Verlauf eines Flusses die hydrologische (z. B. aufgrund großer Zubringer) oder morphologische (z. B. Flusstyp) Charakteristik, so ist eine Abgrenzung auf Ebene der Meso-Skala vorzunehmen. Innerhalb der einzelnen meso-skaligen Flussabschnitte kann nun aufgrund der ähnlichen Charakteristik (z. B. in Bezug auf Form und Größe der betrachteten Hochwasserwelle) ein objektiver Vergleich zwischen den Überflutungsflächen durchgeführt werden, welcher als Grundlage für eine Reihung der Überflutungsflächen dienen kann.

Schließlich bietet die Makro-Skala noch die Möglichkeit, einzelne Flussabschnitte miteinander in ihrer Wirksamkeit $\mathrm{zu}$ vergleichen und großräumige Effekte wie beispielsweise Überlagerungen von Zubringern zu berücksichtigen. Die Makro-Skala kann somit ein wertvolles Werkzeug für Planungen sein, die das gesamte Einzugsgebiet umfassen.

\subsubsection{Hydrologische Parameter}

Unter hydrologischen Parametern werden in diesem Zusammenhang Kenngrößen verstanden, die als Verformung der Hochwasserwelle großräumige (regionale) Bedeutung für das Hochwassergeschehen haben. Hierunter fallen die bereits erwähnte und zweifellos sehr bedeutsame Reduktion des Wellenscheitels $(\Delta Q)$, aber auch die zeitliche Verzögerung der Welle $(\Delta \mathrm{t})$. Letztere ist von großer Bedeutung was die Vorwarnzeiten betrifft, da eine deutlich verzögerte Welle die Reaktionszeit von Einsatzkräften und Anrainern verlängert (z. B. für den Aufbau von mobilen Elementen, die Installation von Objektschutzmaßnahmen, die Räumung von gefährdeten Bereichen etc.). Auf Ebene der Makro-Skala (für den gesamten Flussverlauf oder ganze Flusssysteme) können noch Betrachtungen zu Überlagerungseffekten zwischen den Wellenscheiteln der jeweiligen Zubringer hinzukommen. Für die Beurteilung der Effektivität von Überflutungsräumen mittels der FEM-Methode ergibt sich bei der Betrachtung der hydrologischen Parameter, dass jene Flächen, die eine hohe Scheitelreduktion und/oder eine große Wellenverzögerung aufweisen, als besonders wirksam und daher als besonders erhaltenswert klassifiziert werden.

Die Ermittlung der hydrologischen Parameter erfolgt zuerst auf Ebene der Mikro-Skala und wird dann auf Ebene der Meso-Skala aggregiert um Vergleiche zwischen den Überflutungsräumen zu ermöglichen. Hierfür werden in einem ersten Schritt hydrodynamischnumerische Modelle der einzelnen Überflutungsräume erstellt und diese mit einer der jeweilig zu betrachtenden Jährlichkeit entsprechenden Welle beschickt. Die Differenz zwischen Inputwelle zu Beginn des Modells und Outputwelle an dessen Ende ergibt $\Delta \mathrm{Q}$ und $\Delta \mathrm{t}$ für dieses Gebiet. Zieht man von diesen Werten noch die Flussschlauchretention ab (also die Wirkung, die nur im Flussschlauch erzielt wird, was errechnet werden kann, indem man dasselbe Modelle mit deaktivierter Überflutungsfläche rechnet), so erhält man die reine Rückhaltewirkung des betrachteten Überflutungsraums.

Die Reduktion des Wellenscheitels $(\Delta Q)$ kann schließlich als relative Scheitelabminderung $\left(\Delta \mathrm{Q}_{\mathrm{rel}}\right)$ in Prozent des maximalen Wellenscheitels $\left(\mathrm{Q}_{\max }\right)$ der Inputwelle angegeben werden (Gl. 1): 
Überflutungsraum 1
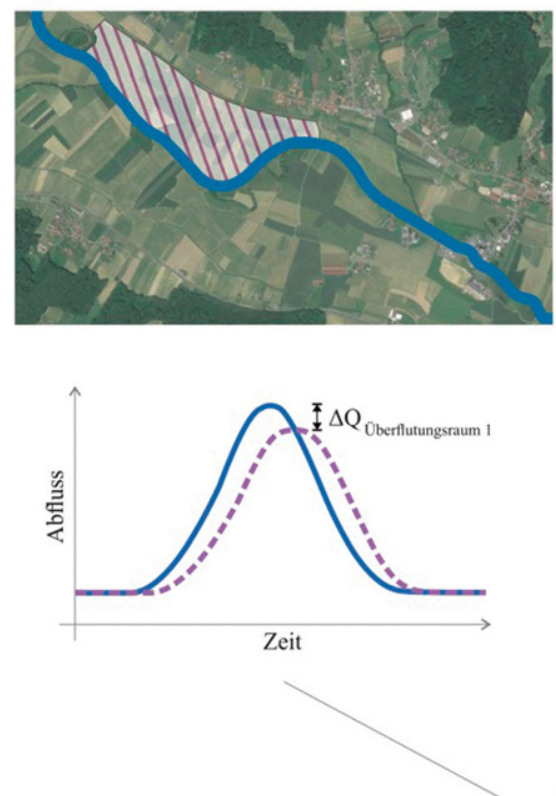

Überflutungsraum 2
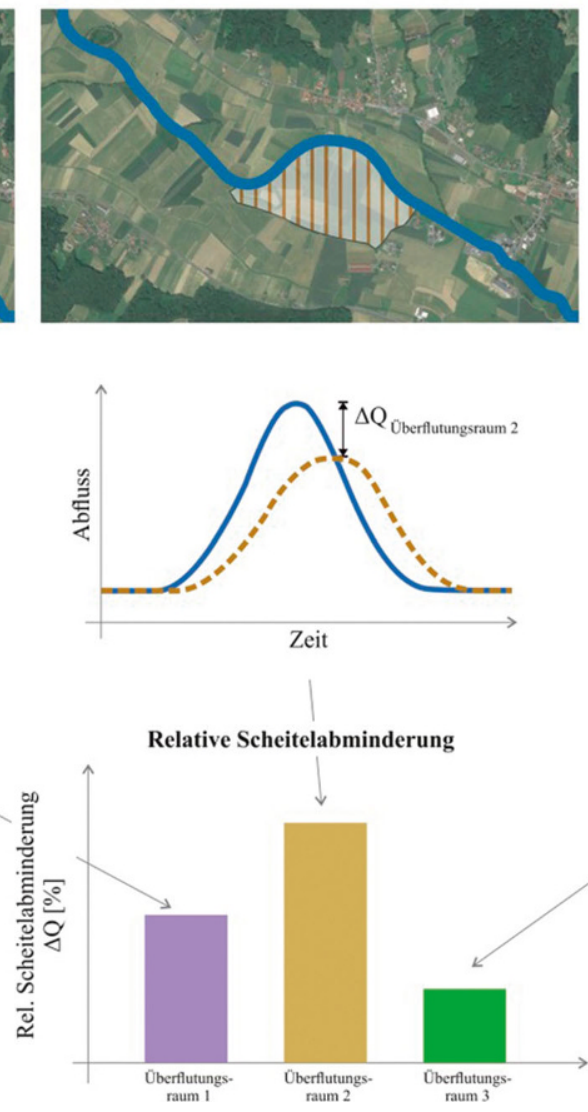

Überflutungsraum 3
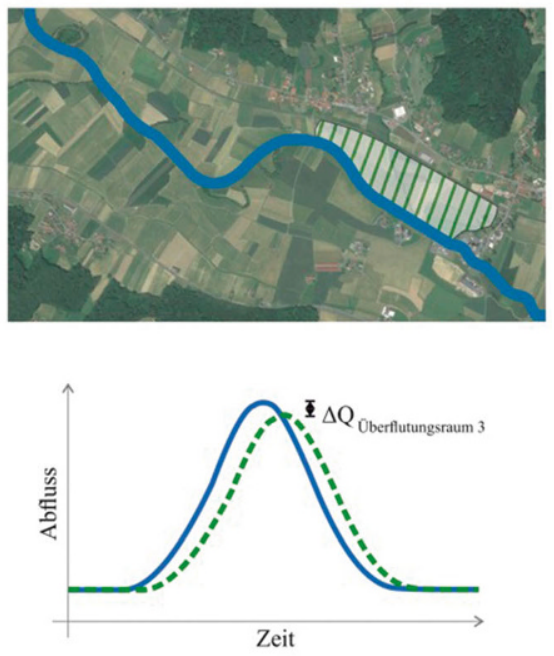

Abb. 2 Exemplarische Ermittlung von $\Delta Q$ für drei ausgewählte Überflutungsflächen

$$
\Delta Q_{\text {rel }}=\frac{\Delta Q}{Q_{\max }} \times 100[\%]
$$

Abb. 2 zeigt exemplarisch die Vorgehensweise für die Ermittlung von $\Delta \mathrm{Q}$ für drei Überflutungsflächen an, an deren Ende die Gegenüberstellung der ermittelten Werte auf Ebene der Meso-Skala steht.

Bei der Ermittlung der zeitlichen Verzögerung der Welle $(\Delta t)$ wird analog vorgegangen. Die Werte für die zeitliche Verzögerung können direkt als zeitliche Differenz zwischen den Wellenscheiteln der Input- und der Outputwelle für jeden Überflutungsraum abgelesen und auf Ebene der Meso-Skala miteinander in Vergleich gebracht werden.

\subsubsection{Hydraulische Parameter}

Bei den hydraulischen Parametern wird der Fokus auf jene Kenngrößen gelegt, die für Belange des lokalen Hochwasserschutzes von Bedeutung sind. Besonders wichtig ist hierbei sicherlich der Parameter Änderung der Wasserspiegellage $(\triangle \mathrm{WSP})$, da Hochwasser- schutzmaßnahmen i.d.R. auf eine bestimmte Abflusshöhe ausgelegt sind. Schon wenige Zentimeter Änderung können über Funktionieren oder Versagen einer Schutzmaßnahme bestimmen. Im Rahmen der hydraulischen Betrachtung können zudem bei Bedarf auch Kenngrößen wie Fließgeschwindigkeiten oder Sohlschubspannungen miteinbezogen werden. Vor allem die Bewertung der Sohlschubspannung ist als wichtige Größe hinsichtlich zukünftiger Erweiterungen in Hinblick auf die FEM-Bewertung von Feststoffund Morphodynamik zu nennen. Für hochwassertechnische Untersuchungen ist jedoch die Änderung der Wasserspiegellage die mitunter bedeutendste Kenngröße.

Für die Ermittlung der Änderung der Wasserspiegellage wird wie folgt vorgegangen: Die Modelle der einzelnen Überflutungsräume, die bereits für die hydrologischen Untersuchungen verwendet wurden, werden in dem Sinne adaptiert, dass die Überflutungsflächen nun um $50 \%$ (also zur Hälfte) oder gar zu $100 \%$ (zur Gänze) deaktiviert werden. Diese Simulation eines hypothetischen Überflutungsraumverlustes (beispielsweise durch Annahme einer fiktiven Dammführung) zeigt auf, welche Überflutungsräume in Hinblick auf derartige Maßnahmen hydraulisch sehr sensitiv, also mit dementsprechend hohen Änderungen der Wasserspiegellage, reagieren würden. Die Berechnung hat so $\mathrm{zu}$ erfolgen, dass die tatsächlichen Abflussverhältnisse korrekt wiedergegeben werden.

Abb. 3 zeigt die Änderung der Wasserspiegellage für einen Überflutungsraum bei $50 \%$ iger bzw. 100\%iger Verkleinerung desselben.

\subsection{Grenzwertsetzung und integrative Zusammenführung in der Floodplain Evaluation Matrix}

Die Multi-Parameter-Betrachtung liefert für jeden Überflutungsraum hydrologische und hydraulische Kennwerte, die aufgrund der Gleichheit der verwendeten Eingangswellen (selbe Jährlichkeit und selbe Wellenform) innerhalb eines meso-skaligen Flussabschnittes 

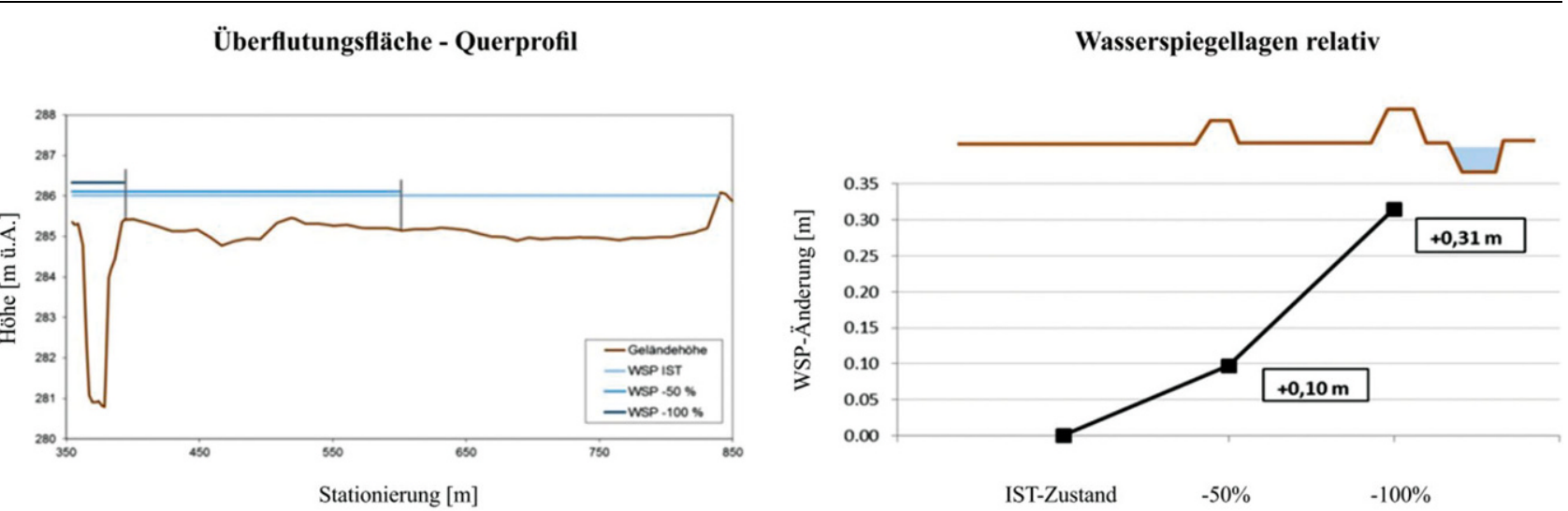

Abb. 3 Veränderung der Wasserspiegellagen bei fiktiven Dammführungen

miteinander vergleichbar sind. Wie bereits im Skalenansatz beschrieben, besteht jeder meso-skalige Flussabschnitt aus bis zu 10 bis 30 Überflutungsräumen, die nun hinsichtlich der einzelnen Parameter $\Delta \mathrm{Q}, \Delta \mathrm{t}$ und $\Delta \mathrm{WSP}$ miteinander verglichen werden können. Die sektorale Reihung der Überflutungsräume für jeden dieser Parameter zeigt, welche Überflutungsräume in Bezug auf den jeweiligen Parameter von besonders großer Bedeutung für diesen Flussabschnitt sind bzw. welche Überflutungsräume nur eine geringere Bedeutung aufweisen. Möchte man die besonders bedeutsamen Flächen ermitteln (z.B. mithilfe des Gefahrenzonenplans, eines Landesraumplans [z. B. Blauzone in Vorarlberg], eines GE-RM Gewässerentwicklungs- und Risikomanagementkonzepts oder Regionalprogramms), so müssen Grenzwerte eingeführt werden, die zwischen den besonders bedeutsamen und den weniger bedeutsamen Flächen differenzieren. Im Rahmen des österreichweiten Projekts FEM-Österreich (Habersack et al. 2014b) wurden aus diesem Grund fünf in ihrer Charakteristik unterschiedliche Flüsse (Inn, Ill, Lavant, Raab, oö. Krems) mithilfe der FEM-Methodik untersucht, um aufbauend auf diesen Daten sinnvolle Grenzwerte zu entwickeln. Die Festlegung von Grenzwerten ist normativer Natur und somit Aufgabe strategischer Planung seitens der EntscheidungsträgerInnen. Wesentlich war die Festlegung österreichweit einheitlicher Grenzwerte, die auf Basis bestehender Abflussuntersuchungen ermittelbar, für Nicht-Fachleute nachvollziehbar und in diversen Verfahren (z. B. Wasserrechts-

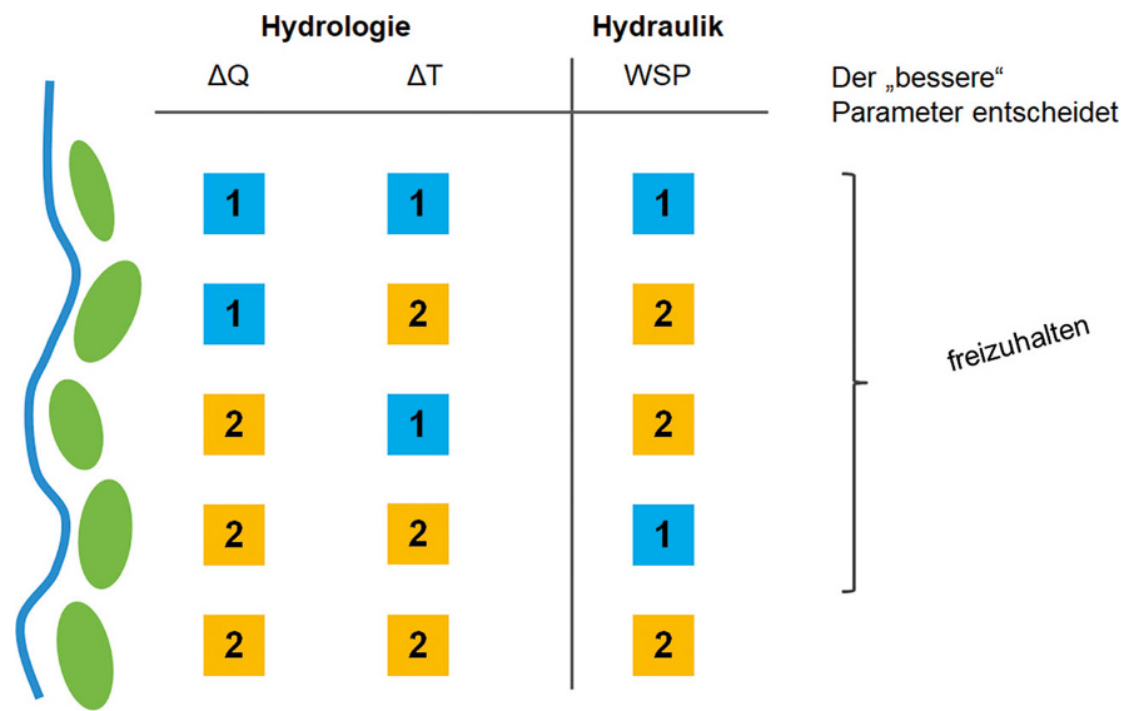

Abb. 4 Gesamtheitliche FEM-Bewertung basierend auf hydrologischen und hydraulischen Parametern

verfahren) einsetzbar sind. Aus dem Projekt ergaben sich folgende Vorschläge, die als sich als praktikabel erwiesen:

\subsubsection{Grenzwert für die Reduktion des Wellenscheitels $(\Delta Q)$}

Um dem Ziel gerecht zu werden, einen möglichst großen Teil der scheitelreduzierenden Rückhaltewirkung eines Flussabschnitts zu erhalten, wurde vorgeschlagen, den etwaigen Verlust an Rückhaltewirkung durch gering wirksame Überflutungsräume mit $5 \% \mathrm{zu}$ begrenzen. Das bedeutet, dass jene bestwirkenden Überflutungsräume, die zusammen $95 \%$ der Rückhaltewirkung erzielen, als „besonders bedeutsam“ klassifiziert werden. Die gesamte Rückhaltewirkung ergibt sich aus der Summe der Wirkungen der einzelnen Überflutungsräume. Die Teilwirkung des jeweiligen Überflutungsraumes im gesamten Flussabschnitt als Prozentwert wird wie folgt errechnet (Gl. 2):

\section{Anteil an der Effektivität eines}

Flussabschnittes für Überflutungsraum ${ }_{i}$

$$
=\frac{\Delta Q_{\text {Überflutungsraum }}}{\sum \Delta Q_{\text {Flussabschnitt }}} \times 100[\%]
$$

wobei

\footnotetext{
$\Delta Q_{\text {Überflutungsraum } i}$ $=$ Reduktion des Wellenscheitels für einen bestimmten Überflutungsraum

$\sum \Delta Q_{\text {Flussabschnitt }}$

= Summe der Reduktionen des Wellenscheitels für alle einzelnen Überflutungsräume eines Flussabschnitts
} 

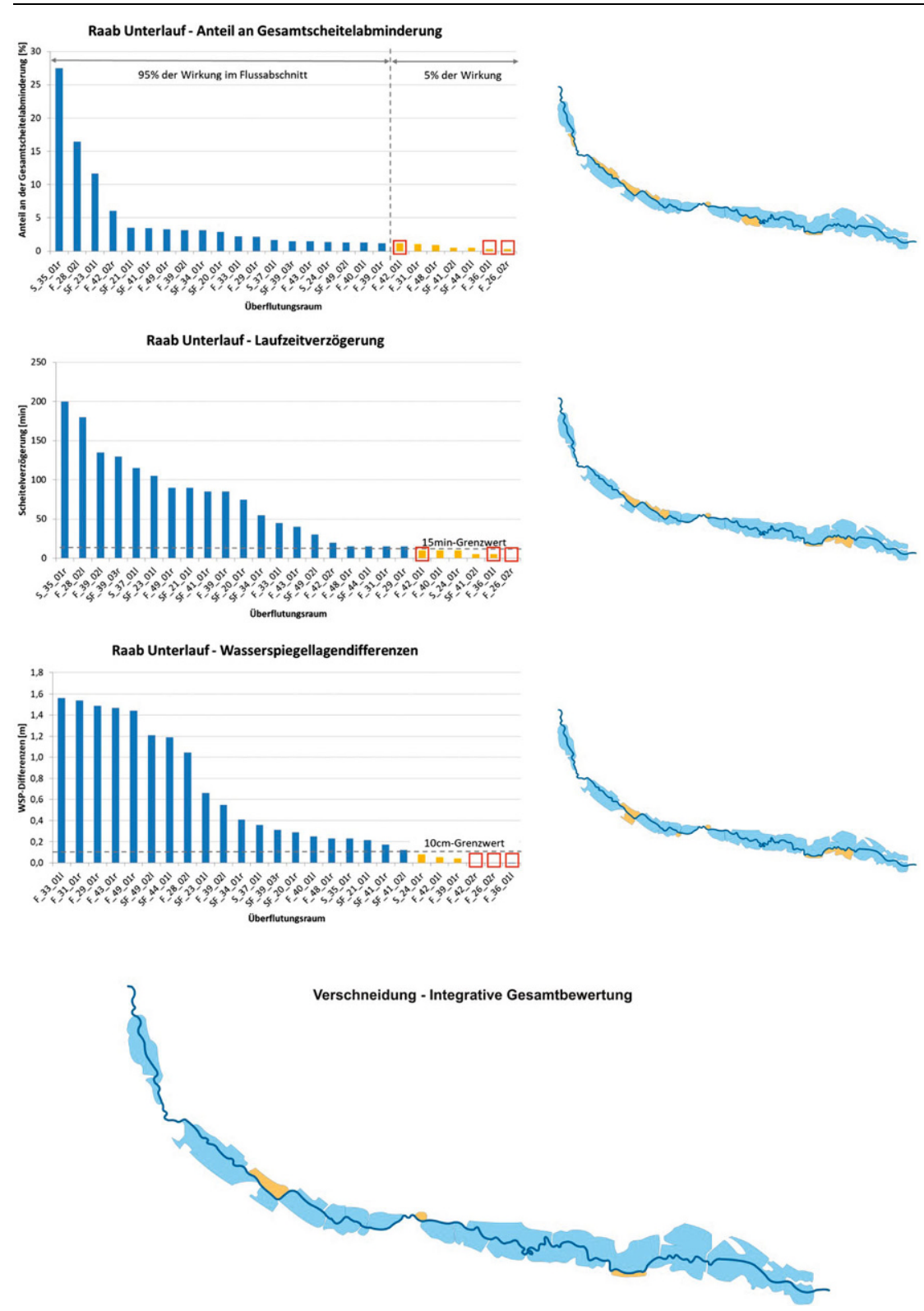

Abb. 5 Integrative FEM-Bewertung am Beispiel der Raab

\subsubsection{Grenzwert für die zeitliche Verzögerung der Welle ( $\Delta t)$}

Die Festlegung des Grenzwerts für die zeitliche Verzögerung der Welle erfolgt anhand der gängigen Zeitschritte bei Pegelaufzeichnungen und hydrodynamisch-numerischen Modellierungen. Erzielt ein einzelner Überflutungsraum eine Verzögerung von mehr als $15 \mathrm{~min}$, so wird dieser als „besonders bedeutsam" in Hinblick auf diesen Parameter definiert.

\subsubsection{Grenzwert für die Änderung der Wasserspiegellage ( $\triangle W S P$ )}

Der Grenzwert für die Änderung der Wasserspiegellage wird mit $10 \mathrm{~cm}$ Wasserspiegelanstieg festgelegt, da eine solche Änderung als bedeutsam für die Wirkung von Schutzmaßnahmen bzw. eine etwaige Schadwirkung erachtet werden kann. Dieser Wert deckt auch den möglichen Ungenauigkeitsbereich des der Modellierung zugrunde liegenden digitalen Geländemodells ab. Jene Überflutungsräume, deren vollständiger (100\%iger) Wegfall eine Erhöhung der Wasserspiegellage um mehr als $10 \mathrm{~cm}$ bewirken würden, werden als sensitiv und damit als „besonders bedeutsam“ für Belange des lokalen Hochwasserschutzes klassifiziert.

\subsubsection{Integrative Zusammenführung}

Bei der integrativen Zusammenführung werden die ermittelten Werte für die einzelnen sektoralen Parameter miteinander verschnitten. Hierbei gilt, dass ein Überflutungsraum insgesamt als „besonders bedeutsam“ zu bezeichnen ist, sobald er bei einem der drei sektoralen Parameter als „besonders bedeutsam“ klassifiziert wurde. Dies bedeutet, dass nur jene Überflutungsräume als insgesamt „gering bedeutsam" eingestuft werden, die bei allen drei betrachteten Parametern eine geringe Wirksamkeit aufweisen (Abb. 4).

\section{Ergebnisse}

\subsection{Fallbeispiel Raab}

Im Folgenden werden anhand der Raab die Ergebnisse der FEM-Methode beispielhaft für einen österreichischen Fluss dargestellt. Die Raab ist ein in der Steiermark und im Burgenland gelegener mäandrierender Tieflandfluss. Das Untersuchungsgebiet für diese Studie ist der steirische Bereich von Flusskilometer 78 bis $19\left(47^{\circ} 15^{\prime} 49.27^{\prime \prime} \mathrm{N} ; \quad 15^{\circ} 34^{\prime} 56.93^{\prime \prime} \mathrm{E}\right.$ bis $46^{\circ} 56^{\prime} 02.39^{\prime \prime} \mathrm{N}$; $16^{\circ} 04^{\prime} 40.72^{\prime \prime} \mathrm{E}$ ). Das $\mathrm{HQ}_{100}$ am Ende des Untersuchungsgebiets beträgt in etwa $300 \mathrm{~m}^{3} / \mathrm{s}$. Im Gebiet konnten 26 Überflutungsräume identifiziert werden, die mithilfe der FEM-Methode in Bezug auf die drei verschiedenen Parameter $\Delta \mathrm{Q}, \Delta \mathrm{t}$ und $\Delta$ WSP untersucht wurden.

Abb. 5 veranschaulicht die Vorgehensweise grafisch. Das oberste Balkendiagramm zeigt die Werte für die Reduktion des Wellenscheitels $(\Delta \mathrm{Q})$. Um $95 \%$ der Gesamteffektivität im Flussabschnitt zu erhalten, müssen die 19 wirksamsten Überflutungsräume erhalten werden. Sieben Überflutungsräume wurden als „gering bedeutsam“ klassifiziert.

Das zweite Balkendiagramm zeigt selbiges für die zeitliche Verzögerung der Welle $(\Delta \mathrm{t})$. Sechs Überflutungsräume verzögerten die Hochwasserwelle um weniger als $15 \mathrm{~min}$ (,gering bedeutsam“), wohingegen 20 Überflutungsräume Werte von über $15 \mathrm{~min}$ aufwiesen („besonders bedeutsam“).

Das dritte Balkendiagramm zeigt die Klassifikation für die Änderung 
Tab. 1 Integrative Zusammenführung der FEM-Methodik für die betrachteten Flüsse (die Zahlen geben die Anzahl der Überflutungsräume an; nicht hinterlegt sind jene, die als „,besonders bedeutsam“, fett sind jene, die als „geringer bedeutsam“ eingestuft wurden)

\begin{tabular}{|l|l|l|l|l|}
\hline Fluss & $\Delta Q$ & $\Delta t$ & $\Delta$ WSP & Gesamt \\
\hline Inn & 35 & 42 & 44 & 56 \\
\hline \multirow{2}{*}{ III } & $\mathbf{2 8}$ & $\mathbf{2 1}$ & $\mathbf{1 9}$ & $\mathbf{7}$ \\
& 11 & 12 & 7 & 14 \\
\hline Lavant & $\mathbf{3}$ & $\mathbf{2}$ & $\mathbf{7}$ & $\mathbf{0}$ \\
\hline Raab & 24 & 22 & 18 & 25 \\
\hline & $\mathbf{7}$ & $\mathbf{9}$ & $\mathbf{1 3}$ & $\mathbf{6}$ \\
\hline & 19 & 20 & 20 & 23 \\
\hline
\end{tabular}

der Wasserspiegellagen ( $\Delta$ WSP), wobei sechs Überflutungsflächen kleinere Änderungen als $10 \mathrm{~cm}$ aufwiesen und somit als „gering bedeutsam“ eingestuft wurden.

Für die integrative Zusammenschau wurden nun jene Überflutungsflächen identifiziert, bei denen alle drei Parameter als „gering bedeutsam“ klassifiziert wurden. Im Beispiel der Raab sind dies drei Überflutungsflächen (in Abb. 5 rot hervorgehoben). Die anderen 23 Überflutungsflächen weisen bei zumindest einem Parameter die Einstufung „besonders bedeutsam" auf und wurden daher auch insgesamt als „besonders bedeutsam" klassifiziert.

\subsection{Weitere Flüsse im Überblick}

Zusammenfassend lassen sich auch die Ergebnisse für die weiteren betrachteten Flüsse auf diese Weise darstellen (Tab. 1). Nach integrativer Zusammenführung ergeben sich mit den zuvor vorgestellten Grenzwerten folgende Ergebnisse: Beim Inn wurden 56 der 63 Überflutungsräume, bei der Ill alle 14 von 14 und bei der Lavant 25 von 31 als „besonders bedeutsam“ ausgewiesen.

\section{Schlussfolgerungen}

Die Erhaltung und - wo möglich die Wiederherstellung - von Überflutungsräumen wird in der EU-Hochwasserrichtlinie (2007/60/EC) als nachhaltige, nicht-technische Hochwasserschutzmaßnahme propagiert, welche vielseitige Vorzüge im Rahmen eines integrierten Flussgebietsmanagements aufweist. Auf der einen Seite können funktionierende Überflutungsräume die Hochwasserwelle dämpfen und somit das Risiko lokal und regional verringern, auf der anderen Seite wird durch den Erhalt von Überflutungsräumen einer Akkumulation von hochwassersensiblen Bebauungen auf gefährdeten Flächen vorgebeugt und somit das Restrisiko verringert. Nichtsdestotrotz findet gerade in Österreich nach wie vor ein starker Flächenverbrauch von ca. 16 ha/Tag statt (Umweltbundesamt 2016), welcher vorwiegend auf Überflutungsflächen stattfindet. Aus diesem Grund wurde mit der Floodplain Evaluation Matrix (FEM) eine Methode entwickelt, die den relevanten EntscheidungsträgerInnen als Werkzeug dienen soll, um die für den Hochwasserschutz besonders bedeutsamen Überflutungsflächen $\mathrm{zu}$ identifizieren und mit geeigneten Mitteln (z. B. Gefahrenzonenplan, Landesraumplan [z. B. Blauzone in Vorarlberg], Gewässerentwicklungsund Risikomanagementkonzept GERM oder Regionalprogramme) freizuhalten. Die mit der FEM ermittelte Prioritätenreihung zeigt, welche Überflutungsflächen eine besonders hohe Wirksamkeit aufweisen und wo demnach das Kosten-Nutzen-Verhältnis für deren Erhaltung besonders hoch ist. In weiterer Folge können diese Ergebnisse auch Grundlage für weitere Maßnahmenplanungen im Zuge eines integrierten Hochwasserrisikomanagements sein. So kann beispielsweise mit der sogenannten "inversen FEM“ der umgekehrte Weg beschritten und ermittelt werden, welche wirkungsmäßigen Kompensationsmaßnahmen bei Errichtung von technischen Schutzbauten erforderlich und realisierbar wären.

Weiters kann durch den prozessbasierten Ansatz und die Trennung in hydrologische und hydraulische Bewertung gewährleistet werden, dass in Zukunft gezielt an die Ziele der notwendigen Sedimentbewirtschaftung von Flusseinzugsgebieten angeknüpft wird. So gibt es bereits erste Ansätze zur
Bewertung und quantitativen Analyse der Retention von Feinsediment in der Fläche (vgl. Hauer et al. 2017).

In Bezug auf die hydraulische Wirksamkeit kann in einer erweiterten FEM der Einfluss von Änderungen der Geometrie (z. B. Anlandungen in Gewässeraufweitungen durch transportierte Sedimente) auf den Wasserspiegel direkt bewertet werden (z.B. Aufspiegelung durch Reduktion des Gewässerquerschnitts) bzw. kann auch die Veränderung des Anspringens des Überflutungsraums durch eine Veränderung von Qbordvoll in einer integrativen Betrachtung analysiert werden. Die FEM bildet also, mit den bestehenden Möglichkeiten zur Erweiterung, eine Grundlage für zukünftige Fragen der Flusseinzugsgebietsbewirtschaftung inklusive der Wasserkraft (z. B. Rückhalt von Sedimenten, die im HW-Fall remobilisiert werden).

In Summe stellt diese Methode eine weitere wertvolle Basis für integrative Hochwasserschutz-Planungen auf Flussabschnitts- und Einzugsgebietsebene im Rahmen eines integrierten Hochwasserrisikomanagements dar.

Danksagung Die Autoren danken dem Bundesministerium für Land- und Forstwirtschaft, Umwelt und Wasserwirtschaft für die Finanzierung des Projekts „FEM-Österreich“ sowie den Bundesländern Vorarlberg, Tirol, Kärnten, Oberösterreich und Steiermark für deren Unterstützung. Die finanzielle Unterstützung durch das Bundesministerium für Wissenschaft, Forschung und Wirtschaft und der Stiftung Forschung, Technologie und Entwicklung wird dankend anerkannt. Außerdem danken die Autoren Julian Hausdorfer für die Unterstützung bei den hydrodynamisch-numerischen Berechnungen und die Bereitstellung von Rechenergebnissen.

Funding Open access funding provided by University of Natural Resources and Life Sciences Vienna (BOKU).

Open Access Dieser Artikel wird unter der Creative Commons Namensnennung 4.0 International Lizenz (http:// creativecommons.org/licenses/by/4. $0 /$ deed.de) veröffentlicht, welche die Nutzung, Vervielfältigung, Bearbeitung, Verbreitung und Wiedergabe in jeglichem Medium und Format erlaubt, sofern $\mathrm{Sie}$ den/die ursprünglichen $\mathrm{Au}$ - 
tor(en) und die Quelle ordnungsgemäß nennen, einen Link zur Creative Com- mons Lizenz beifügen und angeben, ob

Änderungen vorgenommen wurden.

\section{Literatur}

Barredo, J.I. (2007): Major flood disasters in Europe: 1950-2005. Journal of Natural Hazards 42:125-148

Barsch, D., Gude, M., Mäusbacher, R., Schukraft, G., Schulte, A. (1994): Feststoff- und Sedimentdynamik von Elsenz und Neckar bei Hochwasserabfluß. In: Matschullat J, Müller G (eds). Geowissenschaften und Umwelt. Springer, Berlin, Heidelberg

Blackwell, M.S.A., Maltby, E. (2006): Ecoflood Guidelines - How to use floodplains for flood risk reduction. European Commission. Luxembourg Office for Official Publications of the European Communities. 148 pages. ISBN 92-79-00962-1 Bogardi, J.J., Leentvaar, J., Nachtnebel, H.P. (2012): GWSP River Basins and Change. UNES$\mathrm{CO}$

De Kok, J.-L., Grossmann, M. (2010): Largescale assessment of flood risk and the effects of mitigation measures along the Elbe River. Natural Hazards 52 (1):143-166

EU-Hochwasser-Richtlinie (2007/60/EC): Richtlinie über die Bewertung und das Management von Hochwasserrisiken. Europäisches Parlament und Europäischer Rat, Official Journal of the European Union, L 288/27-34, 6.11.2007

Flicker, P., Freiberger, E. (2014): Hochwasserbericht Hochwasser Juni 2013 - Quantitative Bestimmung der Schwebstofffracht längs der österreichischen Donau. BMLFUW, Wien, 29 pp Freshwater, D., Arthur, L.M. (1985): Towards an assessment of the economic impacts of the Canada-Manitoba and Canada-Saskatchewan Flood Damage Reduction Programs. Faculty of Agriculture, University of Manitoba, Occasiona Series 15

Habersack, H., Bürgel, J., Kanonier, A. (2009) FloodRisk II - Vertiefung und Vernetzung zukunftsweisender Umsetzungsstrategien zum integrierten Hochwassermanagement. Wien.

Habersack, H., Hauer, C., Schober, B., Dister, E., Quick, I., Harms, O., Wintz, M., Piquette, E., Schwarz, U. (2008): Flood risk reduction by preserving and restoring river floodplains (PRO_Floodplain) - Final Report. Era-Net CRUE 1st Call. Era-Net CRUE, EU
Habersack, H., Moser, A. (2003): Plattform Hochwasser - Ereignisdokumentation Hochwasser August 2002. Lebensministerium, Vienna Habersack, H., Pucher, K., Schober, B., Samek, R. (2014a): Hochwasser Juni 2013 Ereignisdokumentation. BMLFUW, Wien

Habersack, H., Schober, B., Hauer, C. (2013): Floodplain Evaluation Matrix (FEM) - A novel interdisciplinary method for evaluating river floodplains in the context of integrated flood risk management. Natural Hazards 75: 5-32

Habersack, H., Schober, B., Hauer, C., Wagner B., Fritsch, M., Haspel, H., Ablinger, S., Lagler, S., Nöscher, R., Oder, F., Pollak, M. (2014b): Entwicklung einer Methodik zur integrativen Überflutungsraumbewertung in Österreich. BMLFUW, Wien.

Hauer, C., Obruca, W., Wagner, B., Habersack, H. (2016): Ökologische Bedeutung von Feststoffen und gewässermorphologischen Strukturen im Nahbereich von Kleinwasserkraftwerken Österreichische Wasser- und Abfallwirtschaft, 68/11-12, 545-553

Hauer, C., Habersack, H. (2009): Morphodynamics of a 1000-year flood in the Kamp River Austria, and impacts on floodplain morphology. Earth Surface Processes and Landforms, 34(5): 654-682

Hauer, C., Haimann, M., Holzapfel, P., Holzer G., Boschi, M., Wagner, B., Prenner, D., Fuhrmann, M., Brock, B., Schmalzer, B., Schachner, A., Holzer, G., Leitner, P., Graf, W. \& Habersack, H. (2017): Seeentleerung Gepatsch-Endbericht Studie im Auftrag der TIWAG, 223 pp.

Koirala, S. (2011): Some flood damage estimate of the world. UNESCO-IHE Flood Management Education Platform, http://www.unesco-ihe. org/Flood-Management-Education-Platform accessed on 11th October 2011

Krapesch, G., Hauer, C., Habersack, H. (2011): Scale orientated analysis of river width changes due to extreme flood hazards. Natural Hazards and Earth System Sciences, 11: 2137-2147 Kundzewicz, Z.W., Menzel, L. (2005): Natural flood reduction strategies - a challenge. International Journal of River Basin Management 3 (2):125-131
Messner, F., Meyer, V. (2006): Flood damage, vulnerability and risk perception - challenges for flood damage research. In: Schanze J, Zeman E, Marsalek J (eds) Flood Risk Management: Hazards, Vulnerability and Mitigation Measures, vol 67. Springer Netherlands, pp 149-167

Milliman, J.D., Farnsworth, K.L., Jones, P.D., Xu, K.H., Smith, L.C. (2008): Climatic and anthropogenic factors affecting river discharge to the global ocean, 1951-2000. Global and Planetary Change, 62(3-4):187-194

Penning-Rowsell, E.C., Tunstall, S.M. (1996): Risks and Resources: Defining and Managing the Floodplain. In: Anderson MG, Walling DE, Bates PD (eds) Floodplain Processes. John Wiley \& Sons Ltd., pp 493-533

Pleschko, D, Mühlmann, H, Michor, K. (2017): Gewässerentwicklungs- und Risikomanagementkonzepte als Planungsinstrument für ein integratives Flussraummanagement. Österr Wasser- und Abfallw, https://doi.org/10.1007/ s00506-017-0437-5

Syvitski, J.P.M., Vörösmarty, C.J., Kettner, A.J., Green, P. (2005): Impact of Humans on the Flux of Terrestrial Sediment to the global Coastal Ocean. Science, 308:376-380

Umweltbundesamt (2016): Elfter Umweltkontrollbericht. Umweltsituation in Österreich. Umweltbundesamt, Wien.

Vörösmarty, C.J., McIntyre, P.B., Gessner, M.O. Dudgeon, D., Prusevich, A., Green, P., Glidden, S., Bunn, S.E., Sullivan, C.A., Reidy Liermann, C., Davies, P.M. (2010): Global threats to human water security and river biodiversity. Nature, 467:555-461

Walling, D.E. (2006): Human impact on landocean sediment transfer by the world's rivers. Geomorphology, 79:192-216

Wharton, G., Gilvear, D.J. (2007): River restoration in the UK: Meeting the dual needs of the European Union Water Framework Directive and flood defence? International Journal of River Basin Management 5 (2):143-154 
Hier steht eine Anzeige.

Springer 
Hier steht eine Anzeige.

Springer 